\title{
LA CONFIGURACIÓN DE IDENTIDADES COMO POSICIONES DE SUJETO: ANTIESENCIALISMO Y DIFERENCIA EN JUDITH BUTLER
}

\author{
IDENTITIES CONFIGURATION AS SUBJECT POSITIONS: ANTI-ESSENTIALISM \\ AND DIFFERENCE IN JUDITH BUTLER
}

Leticia Inés Sabsay

Universidad de Buenos Aires, Argentina

\section{Resumen:}

De cara a la problemática en torno de la constitución de identidades y la articulación de la diferencia en el cuerpo social, la frontera principal que ha delineado el campo del feminismo ha sido la que divide a hombres $\mathrm{y}$ mujeres en función de las relaciones de género. A pesar de que tal encuadre cubre diferentes énfasis de acuerdo a las distintas geografías, disciplinas y dentro de ellas, a las diversas tradiciones y corrientes teóricas, sería difícil argumentar en contra del hecho de que, en la práctica, la noción de género ha sido la piedra fundamental para la consagración de la teoría feminista a partir de los años 1960 y 1970s. Sin embargo, la progresiva aparición de diferencias al interior de la supuesta identidad de "la mujer", sean éstas diferencias de clase, etnia, nacionalidad, u orientación sexual, como así la influencia del postestructuralismo, ha dado con la puesta en cuestión de tal homogeneidad y con la reconsideración de la construcción unitaria no sólo de la identidad, sino también de la subjetividad.

\section{Palabras claves:}

Judith Butler, antiesencialismo, sujeto.

\section{Abstract:}

Facing the issue about the constitution of identities and the articulation of the difference in the society, the main frontier which has outlined the field of feminism has divided men and women regarding their gender relationships. Despite the fact that this setting concerns different emphasis in regard to the different geographies, disciplines and inside them, the various traditions and theoretical trends, it would be difficult to argue against the fact that in the practical issue, gender concept has been the cornerstone for the consecration of the feminist theory from $60 \mathrm{~s}$ and 70s. However, the progressive apparition of differences to the inside of the supposed identity of "woman" (these differences are social status, ethnic group, nationality or sexual orientation, as well as the influence of poststructuralism) has questioned the homogeneity and not only has reconsidered the unitary construction of the identity, but also of the subjectivity.

\section{KEY WORD:}

Judith Butler, anti-essentialism, subject. 
De cara a la problemática en torno de la constitución de identidades y la articulación de la diferencia en el cuerpo social, la frontera principal que ha delineado el campo del feminismo ha sido la que divide a hombres y mujeres en función de las relaciones de género. A pesar de que tal encuadre cubre diferentes énfasis de acuerdo a las distintas geografías, disciplinas y dentro de ellas, a las diversas tradiciones y corrientes teóricas, sería difícil argumentar en contra del hecho de que en la práctica, la noción de género ha sido la piedra fundamental para la consagración de la teoría feminista a partir de los años 1960 y 1970s. Sin embargo, la progresiva aparición de diferencias al interior de la supuesta identidad de "la mujer", sean éstas diferencias de clase, etnia, nacionalidad, $\mathrm{u}$ orientación sexual, como así la influencia del postestructuralismo, han dado con la puesta en cuestión de tal homogeneidad y con la reconsideración de la construcción unitaria no sólo de la identidad, sino que asimismo han obligado a rever la aproximación teórica al problema de la constitución de la subjetividad.

En efecto, dentro de las revisiones de la historia del feminismo, es hoy unánime la opinión de que después del desarrollo en torno de la diferencia de género, el feminismo tornó su mirada hacia las diferencias entre las mujeres. Este giro, cuyo auge se vislumbró sobre todo durante los años 1980s, reconoce que la demarcación del género como una construcción social (distinta del sexo) operó y sigue operando como una categoría altamente productiva para poner en evidencia las estructuras sociales de dominación y las relaciones de inequidad entre mujeres y varones. Pero señala que esta división -propuesta como universal-, resulta de la abstracción y el borramiento de otras estructuras sociales de dominación. En otras palabras, si bien este feminismo rescata la categoría de género, al mismo tiempo apunta que la puesta en correlación del objetivo de la igualdad (de derechos) y la noción de diferencia (naturalizada, pero en definitiva histórica y cultural) que esta categoría supone, vuelve oscuras importantísimas diferencias entre las mujeres. La borradura o el relegamiento de la clase social, la etnia, la edad, la nacionalidad, y / u otros ejes de exclusión, resultado de la primacía de los procesos de generización de los sujetos, iguala a las mujeres dentro del género como un actor subalterno, pero en este movimiento, reproduce al mismo tiempo la dominación de un tipo hegemónico de experiencia de las mujeres, a saber las experiencias de la mujer blanca, de clase media, occidental, contrafigura del sujeto varón de la modernidad filosófica que, para estos años ya había sido puesto en crisis también. Obviamente, no se escapa a este reconocimiento de la heterogeneidad al interior de la categoría "mujer", el hecho de que esta crítica es contemporánea de la crisis de los paradigmas modernos y del debate en torno de la posmodernidad. En efecto, esta reflexión se conjuga con la herencia de los paradigmas postestructuralistas, de modo que este paulatino desplazamiento en pos de las des-esencialización del "sujeto mujer", comienza a emparentarse con la idea de que la identidad de género es una construcción "discursiva" encarnada en sujetos que, a la luz de la posmodernidad, se caracterizarán por estar descentrados y mediados por múltiples dispositivos.

A grandes rasgos, esta última línea de pensamiento nos da las coordenadas de la posición que asumirá Judith Butler, quien, enmarcada dentro del ámbito académico en la tradición postestructuralista, se ha convertido en referente ineludible a la hora de discutir cómo se ha producido y cómo se producen los sujetos generizados, con su teoría de la performatividad genérica. La perspectiva de esta autora es que la realidad subjetiva de los actores sociales se configura a partir de la reproducción de normas, que en tanto que prácticas discursivas, materializan de forma performativa una posición de sujeto atravesado por las reglas del género y del sexo, que no son más que instancias de significación autorizadas por la misma repetición. De este modo lo que aparecería como causa de la posición subjetiva en términos de representación e identificación, esto es llegado el caso, el sexo, que determinaría una posición en el contrato de género específica, es en realidad el efecto performativo de las prácticas discursivas inauguradas por ese contrato de género. Desde esta perspectiva, el "sujeto mujer" es radicalmente des-esencializado (no habría desde esta lógica ningún atributo referencial que pudiera figurar como causa de lo que llamamos mujer) y se daría entonces como efecto de discurso.

Para comprender el sentido del planteamiento de Judith Butler es necesario considerar el contexto de las discusiones al interior del feminismo, que a su vez intervienen en los debates en torno del antiesencialismo y la multiculturalidad. Es cierto que a partir de los inicios de la década de 1990, estas dos vertientes se articularán en algunos casos en pos de una noción de la identidad que, a la vez que anti-esencialista, sea capaz de defender las diferencias identitarias de los distintos colectivos que luchan por el acceso a la representación. Sin embargo, al mismo tiempo supondrán una progresiva bifurcación de acuerdo al énfasis que se le otorgue a uno u otro aspecto de la cuestión.

Por un lado, los debates en torno de la multiculturalidad en el marco de la globalización se concentraron en la tensa relación de lo universal con lo particular, en términos políticos, de lo global con lo local, en la reconceptualización de la noción de ciudadanía en vistas de la supuesta multiplicación de las diferencias al interior de las sociedades -el llamado proceso de fragmentación social-, en el problema de los derechos universales y específicos de acuerdo a la particularidad de los grupos involucrados, y en la fragmentación de identidades y las posibilidades para la acción política en este contexto de reconfiguración de lazosidentitarios. Dentro de este universo de análisis, se desarrolló principalmente una positiva visualización de las diferencias y un celebratorio recibimiento de las nuevas identidades sociales o culturales. Las premisas de esta posición son que todas las identidades en juego son merecedoras de reconocimiento y que la diferencia merece ser reivindicada como tal. Contrariando las 
nociones anteriores sobre lo social, en este caso no se trataría de valorar a los actores en tanto que a pesar de sus diferencias, también cuentan con un rasgo de homogeneidad universal como seres humanos y ciudadanos. Se trataría más bien de que la existencia de la diferencia no sea sólo válida o aceptable, sino de que en términos políticos su autoafirmación sea fundamental y necesaria (constitutiva) para la realización de una verdadera democracia. De algún modo, sobre estas bases se asientan las políticas de identidad (identity politics), que desde los años 1980s hegemonizan la forma de la demanda política en los Estados Unidos, así como otras políticas que pretenden dar respuesta a la búsqueda de reconocimiento de lo que se entiende como la propia identidad particular.

De hecho, es en este contexto que dentro de la producción de conocimiento en el marco de las academias, no sólo se abrió el debate dentro del feminismo sino que asistimos tanto al auge de los estudios descriptivos sobre las "nuevas" identidades (muchos de ellos encuadrados dentro de los estudios culturales y la perspectiva etnográfica), como a la revisión histórica de los procesos de colonización, la constitución de los estados nacionales, el surgimiento de los movimientos sociales, los procesos de descolonización, los nacionalismos, la llamada explosión de fundamentalismos y los estados postcoloniales, esta vez a la luz del eje de la noción de identidad.

En cuanto a la segunda vertiente, es decir aquella que pone su acento en el antiesencialismo, ésta mantendrá en cambio, una actitud fundamentalmente escéptica respecto de la configuración, la legitimación y la institucionalización de la identidad y de la diferencia. Heredera del "giro postestructuralista", la toma de partido de esta posición teórica es lógica ya que dentro de este enfoque tanto la identidad como la diferencia no serían otra cosa que construcciones discursivas, y por tanto cristalizaciones del flujo de sentidos sociales que fijarían relaciones dis-simétricas susceptibles de ser desarticuladas a través de "estrategias deconstructivas". Desde este punto de vista todas las identidades, ya sean estas mayoritarias o minoritarias, hegemónicas o subalternas, tendientes a la reproducción del orden social o subvertidoras del mismo, serán conceptualizadas como inherentemente represivas. En efecto, parafraseando a Nancy Fraser, ellas necesaria y lógicamente implican "una normativa" para los individuos que se identifiquen con el grupo que opera como soporte de esa posición De esta forma, todo establecimiento de una diferencia, o dicho de otro modo, todas las diferencias serán analizadas como constitutivamente excluyentes.

Ahora bien, esta división que trazáramos de comienzo puede resultar algo tramposa si no matizáramos esos términos, que por ánimo de síntesis pueden resultar algo taxativos. Y esto es así porque la noción antiesencialista de la identidad no se ha opuesto tout court a los discursos que apuestan por el reconocimiento de las distintas identidades y en general, de la diferencia. Por el contrario, el discurso antiesencialista que entiende a la identidad como una posición arbitraria y relacional dentro de un juego de diferencias, ha sido profusamente utilizado para defender las diferencias de género y desde ya la diferencia cultural. Es necesario en este sentido insistir en que no se trata de afirmar que tanto los debates en torno de la especificidad cultural como de la diferencia de género hayan recurrido a un discurso esencializante de la identidad. Antes bien, lo que se quiere poner de relieve es que en la interpretación que el discurso de la diferencia ha hecho de la no esencialidad de la identidad colectiva o del sujeto, éste se ha visto obligado a acentuar sólo algunos aspectos del antiesencialismo y ha debido dejar de lado otros, ya que la hora de pensar y llevar hasta las últimas consecuencias la doble necesidad del reconocimiento de la diferencia y la puesta en cuestión de esa misma diferencia a la vez, lo llevaría a posiciones dilemáticas de difícil resolución.

En efecto, ¿cómo defender la especificidad de un colectivo a la hora de luchar por su representación en el espacio público (sea en el campo de la política, de la cultura, de los media, o del arte) y al mismo tiempo poner en cuestión dicha especificidad, desde el momento en que se acepta que la diferencia que traza la frontera de lo "específico" es arbitraria, y no se sostiene en ningún fundamento anterior (natural o trascendental), sino en la historia de las construcciones sociales y culturales siempre atravesadas por el poder? Este es el problema teórico-político que nos planteamos, y a través del cual quisiéramos considerar la propuesta de Judith Butler, quien claramente intenta sortear esta contradicción.

Nuestro punto de partida es que tal vez esta bifurcación o corrimiento entre el antiesencialismo más escéptico y aquél que celebra la diferencia, deba su posibilidad a que la identidad como una formación no esencial basada en los posicionamientos relacionales, parecería albergar dos dimensiones posibles de la identidad como una formación no esencial, que no necesariamente son consistentes entre sí, y que incluso pueden derivar en concepciones radicalmente distintas. Nos referimos por un lado a la noción de identidad como una figura oposicional/relacional, y por el otro a la noción de diferencia como la marca de una posición no clausurada. "El infinito juego de las diferencias" que invocara Derrida (1989), en efecto remite no sólo a la articulación de valores diferenciales, sino asimismo a la iterabilidad de esos valores (Derrida, 1997) La differance es diferencia, pero -como sucede emblemáticamente en la escritura- ésta debe ser entendida también como aplazo, diferimiento, y en este sentido, constitutiva apertura del juego de la significación. En el caso de la teoría de la performatividad genérica, la toma de partido de Butler a favor de la noción derridiana de diferencia, que involucra a su vez a la de iteración, es clara a este respecto: “(L)a performatividad no puede entenderse fuera de un proceso de iteración, un proceso de repetición regularizada y obligada de normas. Y no es una repetición realizada por un sujeto esta repetición es la que habilita al sujeto y constituye la dimensión temporal de ese 
sujeto." (Butler, 2002: 145). Es decir que la iterabilidad de la repetición que hace a la performatividad del género marca los dos aspectos nodales de la noción de diferencia, a saber que ella se monta sobre "lo ya escrito", regulado, diferenciado, delimitado, restringido, pero que en ese proceso de repetición que desplaza y difiere la misma regulación, es que la hace significar algo que nunca puede saberse de antemano.

Es así que por un lado tenemos una noción de identidad basada en lo que definiríamos como la forma antiesencialista relacional. El antiesencialismo de esta forma, en la que subyace la idea de "diferencialidad" y de posicionalidad, está justificado por cuanto esta forma conlleva en primera instancia la definición de la identidad como algo distinto de la pura presencia de valores autosuficientes. Desde esta conceptualización, la definición de una posición identitaria, de hecho, nunca podría ser el resultado de una cadena de atributos inmanentes, cuya fuente fuera un ente o sujeto -a nivel del significadodel que puedan predicarse ciertas características. Antes bien, tributaria de la idea estructuralista de las relaciones formales entre los términos, ésta supone, retomando la herencia saussuriana, la definición de las lógicas identitarias en función de valores oposicionales, es decir en su relación de diferencia con otras posiciones identitarias, en un juego de presencias y ausencias. En efecto, hoy es de sentido común afirmar que no hay nada en los actores sociales en sí que permita situarlos en el espectro social de forma fija, y que al contrario éstos son el producto de sus relaciones diferenciales con otros actores sociales o grupalidades. Podría decirse que es esta noción la que ha nutrido desde hace tiempo ya muchos análisis que desde la perspectiva de género han focalizado en la configuración de lo femenino como otredad y/o particularidad, y más aún, sobre la construcción de todo otro como constitutiva del sí mismo. Pero esta versión oposicional /relacional de la diferencia parecería resultar insuficiente a la hora de realizar un análisis crítico de las relaciones de poder y proceder a la deconstrucción de las identidades fijas en tanto que cristalizaciones normativas.

Es aquí donde aparece esa otra forma del antiesencialismo, que denominaríamos como una forma antiesencialista radical, y en la que ubicamos la conceptualización de la performatividad de Judith Butler, producto de su lectura de Derrida. Esta forma no se agota en la diferencia relacional, sino que incluye asimismo la idea de no clausura: una diferencia que reclama la apertura a la que estaría sujeta toda posición identitaria, en el sentido de que ésta no sólo no podría ser idéntica a sí misma, sino que tampoco podría guardar una relación isomórfica con ningún significado estable en función de sus diferencias con otros significantes. La posición (performativa) de sujeto, como efecto del juego de representaciones e identificaciones, es una posición sometida a la lógica de la diferencia radical que supone la inestabilidad de la iteración de los significantes. En palabras de Judith Butler: “(L)as identificaciones corresponden a la esfera imaginaria... son la sedimentación del "nosotros" en la constitución de cualquier "yo", la presencia estructurante de la alteridad en la formulación misma del "yo". Las identificaciones nunca se concretan plena y finalmente; son objeto de una incesante reconstitución y, como tales, estás sometidas a la lógica volátil de la iterabilildad." (Butler, 2002: 159)

En esta línea de pensamiento, y en sintonía con la teoría de Judith Butler que desarrollamos a lo largo del trabajo, también otras autoras como Chantal Mouffe, desde la teoría política, o Joan Scott, desde el campo de la historia, señalan con claridad esta limitación de los significantes de la identidad con respecto a los significados que pretenderían agotar: "Se asume que las personas son discriminadas porque son ya diferentes, cuando en realidad... es al revés: la diferencia y la aparición de identidades diferentes son producidas por la discriminación, un proceso que establece la superioridad, lo típico, o la universalidad de algunos en términos de la inferioridad, lo atípico, la particularidad de otros" (Scott, 1992: 14, mi traducción). Las palabras de Joan Scott resumen de manera formidable algunos aspectos que es necesario considerar respecto de lo que hemos llamado la forma antiesencialista radical de conceptualizar la identidad. Una conceptualización que señala la imposibilidad de la completud del significante de la identidad no sólo en base a la figura de lo oposicional, sino atendiendo a la imposibilidad de la clausura de la "identidad" en tanto que posición de sujeto. En primer lugar, como lo habíamos señalado renglones más arriba, en esta definición se vuelve a insistir en que la identidad no es el espacio prefijado de algo dado, la expresión de un "ser", sino una construcción que se da en relación a otros. Hasta aquí ambas formas de antiesencialismo -el oposicional, y el radical- coinciden. Pero lo que señala esta afirmación es que el gesto fundante de los posicionamientos identitarios no está en los valores oposicionales de cada grupo (la inferioridad / superioridad, la universalidad / particularidad de unos y otros), sino en la práctica misma de la producción de la frontera, de la discriminación.

Esta práctica de instauración de fronteras puede leerse en clave lingüística, como un efecto de enunciación -y en este sentido, una alocución en la que se instalan las primeras figuras de un "yo" y un "tú"- (Benveniste, 1991) y que consecuentemente entonces se postula como una posición discursiva no clausurada. Justamente lo que queremos señalar es que cuando se piensa en el valor diferencial, no sólo se nos indica que "el prolífico mundo de las diferencias" es un universo que se organiza jerarquizadamente, en pocas palabras, que la diferancia siempre connota un valor (Derrida, 1971). Desde esta perspectiva, se hace referencia también y fundamentalmente a la dis-simetría entre los términos a partir de su iterabilidad y no en función de valores opositivos; al contrario de esta última, la definición derridiana incorpora la alteridad constitutiva del signo asociada a la dimensión temporal. 
Así como sucede con los sentidos de un texto y las posiciones de enunciación que se producen y circulan en y entre los discursos, que permanecen por definición inherentemente abiertos, dentro de esta línea de pensamiento, la conceptualización de los lazos identitarios supondrá necesariamente la instancia de la "no clausura". La constitución de la identidad subjetiva sería en este sentido contingente y en definitiva el producto inestable de múltiples construcciones de sentido, siempre abiertas y prestas al desplazamiento (Mouffe, 1992). Siguiendo la línea de Mouffe, bastante en sintonía con la de Butler y Scott, diríamos que la identidad subjetiva se daría como un momento constituyente que no obstante, o más bien al contrario, justamente debido a su dimensión temporal, estaría signada por la incompletud, esto es "sujeta a una permanente constitución". La clausura que tal momento supone sería el resultado de las permanentes negociaciones que posibilitan y a la vez condicionan la fuerza o la eficacia de una pluralidad de construcciones contradictorias.

En el marco de esta articulación se comprende también que la identidad subjetiva operada a través de esta clausura implique siempre la exclusión. Y es a partir de aqui que podría decirse que la clausura imaginaria que implica la asunción de una posición de sujeto determinada, siempre temporal y precariamente, llama en este sentido a la idea del "falso reconocimiento" (méconaissance) ya que reconocerse en el imaginario como un yo / un nosotros, en tanto que totalidad, es el producto de un borramiento de la inconmensurabilidad del sujeto. En otras palabras, implica negar la imposibilidad del sujeto de reconocerse en la completud de sí mismo, en la medida en que esa completud no es tal, sino el efecto de su constitución a partir del Otro (Lacan, 1970).

Creemos que esta doble articulación del concepto de identidad subjetiva como efecto diferencial y como instancia de no clausura -articulación ésta que discutimos a la luz del pensamiento de Judith Butler- se torna particularmente evidente en el campo de la sexualidad. Podría pensarse que esta precariedad de las categorías identitarias y las metáforas de los tránsitos, como así las llamadas subjetividades posmodernas (que tal vez no sean algo distinto de formas contemporáneas de encarnar y comprender -es decir, de otorgar sentido- a estos desplazamientos políticos), se materializa de forma ejemplar en las luchas actuales por la definición de posiciones cuya orientación e identificación sexuales parecerían poner en cuestión la capacidad de la categoría de género para abordarlas con exhaustividad. Más allá de la categoría de género, y en ciertos casos hasta poniendo en crisis la suficiencia de la misma como criterio analítico, el intento de identificar categorías para la demarcación de un diagrama de posiciones de sujeto posibles que exceda el binarismo mujer / varón, incluyendo otros aspectos de la sexualidad, ofrece, desde el inicio, algunas ambigüedades, y consecuentemente la oportunidad para la problematización teórica sobre la identidad subjetiva y la elaboración de la subjetividad. A nivel analítico, en principio, opera en torno de esta campo, la indeterminación de criterios mediante los cuales establecer las fronteras: además del género, o conjugadas con él, se trataría de la orientación sexual, de la identificación sexual, y / o de las prácticas sexuales, y en cuanto a estas últimas, se trataría de prácticas efectivamente continuadas a lo largo del trayecto de vida?, cómo incluirían estas definiciones pragmáticas la dimensión de la fantasía y los procesos de identificación? Y si todas estas dimensiones intervienen, cómo es que se articularían?

A decir verdad, las luchas teóricas y políticas por la "nominación" ponen al descubierto este estado de precariedad y de inestabilidad que en realidad esconde cualquier categoría que pretenda abarcar completamente una identidad, incluyendo por supuesto la genérica. $Y$ es precisamente esto lo que viene a poner en evidencia la noción performativa del género (cuyos principios analíticos en realidad pueden hacerse extensible a otras formaciones de la identidad basadas en otros ejes como por ejemplo el de la etnicidad). En el caso de las posiciones de sujeto contra-heteronormativas, cuando éstas toman los referentes de la cultura heterosexual para realizarse como tales, rearticulando sus prácticas (las heterosexuales), y en este sentido resignificándolas en su misma repetición, desnudan la ausencia de un anclaje original o sustantivo de las mismas posiciones de género normativizadas dentro de la heterosexualidad (obligatoria), un anclaje que pudiese ser fundado más allá de la propia representación. Ciertamente, uno de los principales objetivos de Butler en El género en disputa, consiste en realizar una crítica antisustancialista del género. Allí, cuando la autora señala que la relación binaria, disyuntiva y asimétrica de los géneros puede ser intervenida justamente por las prácticas paródicas, lo que remarca precisamente es la insustancialidad de las posiciones determinadas por la normativa genérica: "La repetición de constructos heterosexuales dentro de las culturas gay y hétero bien puede ser el sitio inevitable de la desnaturalización y la movilización de las categorías de género; la reproducción de estos constructos en marcos no heterosexuales pone de relieve el carácter totalmente construido del supuesto original heterosexual. Así, gay no es a hétero lo que copia a original, sino más bien, lo que copia es a copia. La repetición paródica de "lo original" (...) revela que esto no es sino una parodia de la idea de lo natural y original" (Butler, 2001: 65).

Prácticas que ponen de relieve los vínculos sinuosos entre las representaciones, las identificaciones, las identidades y las posiciones de sujeto, y que consecuentemente ponen en escena la realidad imaginaria del yo. Lo que nos interesa poner de relieve es que en el caso de las identidades "trans", o de modo más general, en el caso de las subculturas contra-hetero-normativas, lo que se pone de manifiesto en las luchas por la "nominación" y en todas las categorías y subcategorías que proliferan a medida que más y más diferencias van encontrando en el arco identitario alguna posición es el hecho de que a diferencia de otros diagramas más institucionalizados y por 
ende más estables, el de las "identidades sexuales" que se hallan por fuera de lo canónicamente representable por el sistema de sexo / género parecería estar en pleno proceso de lucha política por la institucionalización. Dicho en otras palabras, y de cara a las luchas actuales por el reconocimiento de los derechos de las (mal) llamadas "minorías sexuales", la definición de posiciones de sujeto sexualizadas en el contexto contemporáneo parecería estar expresando justamente la imposibilidad de cierre (de toda identidad) ya que, a diferencia de otras categorías quizás ya más organizadas e institucionalizadas dentro de los sistemas de jerarquías, ésta se encuentra sujeta a la inestabilidad de las luchas por la categorización -en muchos casos todavía no definidas.

En síntesis, la crisis de los lazos identitarios y aun de la misma noción de identidad encuentra una expresión radical en el campo de las sexualidades. La conjugación de la noción de género con las de orientación e identificación sexuales da cuenta de ello. De hecho, el problema de los alcances de la categoría de género como perspectiva analítica para dar cuenta de los mecanismos de exclusión propios del orden sexual hetero-normativo es de hecho uno de los puntos centrales en torno de los cuales se estructura el debate entre la teoría feminista y la teoría queer (Weed \& Schor, 1997).

La somera descripción de las dificultades que acarrea esta articulación (patente en el caso de las categorías que evocan las posiciones "trans" por ejemplo, pero que tal como se ha venido atestiguando en nuestro contexto contemporáneo marcado por la globalización, también puede observarse en relación con el dispositivo conceptual de la "identidad cultural" -tan extendido a partir del crecimiento de los estudios culturales y de las nuevas políticas de localización dadas la reconfiguración de las fronteras geográficas y económicas, las migraciones y las guerras), señala la necesidad de delinear un análisis de la producción dialógica de la identidad -atendiendo a los mecanismos materiales y simbólicos de representación y a los procesos de reconocimiento que hacen posible su materialización en una subjetividad. Poniendo el acento en las experiencias contemporáneas de la indeterminación y la multiplicidad, antes que obliterar la problematicidad de la nominación, recurriendo a un culturalismo simplista basado en una supuesta transparencia del dato, que daría como resultado un abanico de "nuevas" identidades proliferantes y diversas, pero no por ello menos fijas y reificadas, el desafío consiste en hacer visible cuan dificultoso y polémico puede ser el uso teórico de las categorías y su articulación en los sujetos.

En los términos de Butler, se trataría de apostar por la diseminación en la diferencia, lo que implica una reformulación de lo "multi" no ya como la yuxtaposición o sumatoria de posiciones de sujeto, sino como espacio de conformación de un nuevo tipo de subjetividad. Es cierto que esta opción obviamente cuestiona profundamente las posibilidades teóricas -y aun políticas- tradicionalmente basadas en la categorización de identidades estables. Pero también es cierto que ésta es una elección que no podríamos obviar. Nos quedan dos opciones: seguir reproduciendo una normativa cuya pregonada universalidad esconde en realidad un acto de violencia y dejar en la invisibilidad ciertas relaciones de poder, o por lo menos, dar cuenta de esos actos de violencia y sincerarnos con nuestra propia dificultad. En ningún caso se trata de una teoría paralizante de los indecidibles, sino más bien de la politización de las construcciones de la identidad. En efecto, la crítica de las categorías no nos lleva a la imposibilidad de pensar mayores marcos de libertad. Antes bien, es lo que nos habilita no sólo a desarticular las ideologías discriminatorias, sino asimismo, y de cara a esta contradictoria situación, es lo que nos obliga a asumir que no basta con dar cuenta de las diferencias culturales y sexuales, si al mismo tiempo no se critican los mecanismos que hacen posible y organizan jerarquizadamente el acceso a la representación (Zizek, 1998).

En este sentido, si bien el debate aquí esbozado se encuadra principalmente dentro del ámbito de la representación de la sexualidad, la discusión sobre estas figuraciones y las subjetividades que ellas performativamente conforman, tiene por objeto llamar la atención sobre la necesidad de una seguir reflexionando sobre las posibilidades y desafíos que suponen para el analista social estas imágenes que desarticulan una visión naturalista o historicista de la identidad, considerando sobre todo las condiciones de producción que delimitan dentro del mismo ámbito académico las formas de representabilidad posibles para unos sujetos sociales, que si bien son incluidos y bienvenidos en ámbitos académicos y artísticos, como emblemas de la indeterminación, siguen siendo sistemáticamente excluidos y dificultosamente pensables en el imaginario de la ciudadanía, donde en definitiva se juega no sólo la viabilidad de su representación política, sino más apremiante aún, su supervivencia.

\section{REFERENCIAS BibLIOGRÁFICAS}

Benhabib, S. y Cornell, D. (Comps.), Teoría feminista / Teoría crítica, Valencia, Editorial Alfons El Magnánim, 1989.

Benveniste, E., Problemas de lingüística general, México, Siglo XXI, 1991

Butler, J., El género en disputa. El feminismo y la subversión de la identidad, México, Paidós, 2001

----, El grito de Antígona, Barcelona, El Roure, 2001

----, Mecanismos psíquicos del poder. Teorías sobre la sujeción, Madrid, Cátedra, 2001b.

----, Cuerpos que importan. Sobre los límites materiales y discursivos del "sexo", Bs. Aires, Paidós, 2002.

Butler, J., Laclau, E. y Zizek, S., Contingencia, hegemonía y universalidad. Diálogos contemporáneos en la izquierda, Buenos Aires, FCE, 2003.

Derrida, J., “La Differance”, en AA VV/ Grupo Tel Quel, Teoría de Conjunto, Barcelona, Seix Barral, 1971 ----, Márgenes de la filosofía, Madrid, Cátedra, 1989. 
----, El tiempo de una tesis. Deconstrucción e implicaciones conceptuales, Barcelona, Anthropos, 1997.

Lacan, J., Las formaciones del inconsciente, Buenos Aires, Nueva Visión, 1970.

Mouffe, C., "Citizenship and political identity”, October Nro. 61, Cambridge, MIT, Summer 1992

----, "Feminismo, ciudadanía y política democrática radical", Revista de Crítica Cultural, núm. 9, Noviembre, 1994.

Scott, J. W., "Multiculturalism and the politics of identity", October Nro. 61, Cambridge, MIT Press, Summer 1992.

Spivak, G. C., "Can the subaltern speak?", en Nelson C. \& Grossberg, L. (Eds.), Marxism and the Interpretation of Culture, Urbana, Univ. of Illinois Press, 1988.

Weed, E. y Schor, N. (Eds.), Feminism meets queer theory, Bloomington, Indiana Univ. Press, 1997.

Zizek, S. y Jameson, F., Estudios Culturales. Reflexiones sobre el multiculturalismo, Buenos Aires, Paidós, 1998. 